J. Nonlinear Var. Anal. 2 (2018), No. 2, pp. 219-228

Available online at http://jnva.biemdas.com

https://doi.org/10.23952/jnva.2.2018.2.08

\title{
EFFECTIVE STRONG CONVERGENCE OF THE PROXIMAL POINT ALGORITHM IN CAT(0) SPACES
}

\author{
LAURENŢIU LEUŞTEAN ${ }^{1,2,4, *}$, ANDREI SIPOŞ Ş,4 $^{3,4}$ \\ ${ }^{1}$ The Research Institute of the University of Bucharest (ICUB), University of Bucharest, \\ Bd. M. Kogălniceanu 36-46, 050107, Bucharest, Romania \\ ${ }^{2}$ Faculty of Mathematics and Computer Science, University of Bucharest, \\ Academiei 14, 010014, Bucharest, Romania \\ ${ }^{3}$ Department of Mathematics, Technische Universität Darmstadt, \\ Schlossgartenstrasse 7, 64289 Darmstadt, Germany \\ ${ }^{4}$ Simion Stoilow Institute of Mathematics of the Romanian Academy, \\ Calea Griviţei 21, 010702 Bucharest, Romania
}

\begin{abstract}
We apply methods of proof mining to obtain uniform quantitative bounds on the strong convergence of the proximal point algorithm for finding minimizers of convex, lower semicontinuous, proper functions in CAT( 0$)$ spaces. Thus, for uniformly convex functions, we compute rates of convergence, while, for totally bounded CAT(0) spaces, we apply methods introduced by Kohlenbach, Leuştean and Nicolae to compute rates of metastability.
\end{abstract}

Keywords. Proximal point algorithm; CAT(0) spaces; Proof mining; Rates of convergence; Rates of metastability.

2010 Mathematics Subject Classification. 46N10, 47J25, 03F10.

\section{INTRODUCTION}

Let $X$ be a complete CAT( 0$)$ space and let $f: X \rightarrow(-\infty,+\infty]$ be a convex, lower semicontinuous (lsc), proper function that has at least one minimizer. In analogy to the celebrated notion in Hilbert spaces, Jost introduced [7] in 1995 the resolvent $J_{\gamma}$ of $f$ of order $\gamma>0$ as

$$
J_{\gamma}: X \rightarrow X, \quad J_{\gamma}(x)=\operatorname{argmin}_{y \in X}\left[f(y)+\frac{1}{2 \gamma} d^{2}(x, y)\right] .
$$

It may be shown that when $f$ satisfies the conditions specified above, the argmin of the right hand side is well-defined, i.e., it exists and is unique.

Resolvent operators corresponding to diverse forms of objects had already been defined and applied to great success in the field of convex optimization in linear settings. The main tool used to exploit them, developed by Brézis and Lions [3], Martinet [18], and Rockafellar [19], was the proximal point algorith$m$, and for a general introduction to this field in the context of Hilbert spaces, putting this algorithm in its context, one may see the book of Bauschke and Combettes [1].

${ }^{*}$ Corresponding author.

E-mail addresses: laurentiu.leustean@unibuc.ro (L. Leuştean), sipos@ mathematik.tu-darmstadt.de (A. Sipoş).

Received January 7, 2018; Accepted February 16, 2018.

(C)2018 Journal of Nonlinear and Variational Analysis 
This algorithm was first proven to work in the setting of complete CAT(0) spaces by Bačák [2], who showed some years ago its weak convergence (that is, $\Delta$-convergence). Specifically, in the above context, if one takes a starting point $x \in X$, and a sequence of step-sizes $\left(\gamma_{n}\right)_{n \in \mathbb{N}}$, then one may define the proximal point algorithm $\left(x_{n}\right)_{n \in \mathbb{N}}$ by setting:

$$
x_{0}:=x, \quad x_{n+1}:=J_{\gamma_{n}} x_{n} \text { for any } n \in \mathbb{N} .
$$

It is of this sequence that Bačák proved the weak convergence to a minimizer of $f$, assuming additionally that the series $\sum_{n=0}^{\infty} \gamma_{n}$ is divergent.

It is interesting to ask whether the algorithm may converge in the usual sense (i.e., strongly). Although this is known not to hold in general (see [6, Corollary 5.1] for a counterexample in the context of Hilbert spaces), Bačák selected two cases where it can be guaranteed to happen. This is expressed by the following result (cf. [2, Remark 1.7]).

Theorem 1.1. In the above hypotheses, assume further either that $f$ is uniformly convex or that $X$ is locally compact. Then $\left(x_{n}\right)$ converges strongly to a minimizer of $f$.

Our goal in this paper will be to prove a quantitative version of the above Theorem 1.1, as seen through the lens of proof mining.

Proof mining is an applied subfield of mathematical logic (see [8] for a comprehensive introduction and $[9,10]$ for a survey of recent results), whose aim is to obtain quantitative information - primarily witnesses and bounds for existentially quantified variables - out of ordinary mathematical proofs that cannot be said to be fully constructive. For example, in the case above, where the conclusion of the proof is a convergence statement, the relevant piece of quantitative information would be a formula that is, a function of low computational complexity - yielding, for a given $\varepsilon$, the rank from which all terms of the sequence are closer than $\varepsilon$ to the desired limit. (In this case, a bound will also be a witness, by the monotonicity of the statement.) Unfortunately, fundamental results in computability theory lead to the impossibility of such a formula - called a rate of convergence - in relatively simple contexts (e.g. ergodic averages), and therefore the most general metatheorems of proof mining prohibit it from the start by restricting the logical complexity of the formulas that may serve as a conclusion to a proof which is under analysis, if that proof makes use of the law of excluded middle. A significant part of the recent research in the field has been devoted to finding methods of 'circumventing' this fundamental limitation.

The two cases of the theorem under discussion shall be analyzed separately, as one deals with the problem above in different ways. The case where $f$ is uniformly convex is a particularly interesting one. It is known that this property immediately yields the uniqueness of the minimizer, and past work of Kohlenbach [11] and Briseid [4] has shown that in this case one may obtain convergence of the sequence in a completely constructive way, and hence extract a full rate of convergence out of that argument. Here, we reason more directly, in the sense that we can simply get out of Bačák's own argument the needed rate of convergence for the algorithm in this 'uniform' case. This is what we do in Section 3. We mention that such a rate for a quite general uniformity condition (though not including the case examined here) was previously obtained in the paper [15].

In the second case, when $X$ is locally compact, things are not this simple, as excluded middle features prominently in the proof and one cannot so easily eliminate it. One then works with a related notion of metastability - introduced by Tao in [20] and used extensively in his proof of the convergence theorem for multiple ergodic averages [21] - a property equivalent to that of a sequence being Cauchy. This 
property is expressed as follows:

$$
\forall k \in \mathbb{N} \forall g: \mathbb{N} \rightarrow \mathbb{N} \exists N \in \mathbb{N} \forall i, j \in[N, N+g(N)]\left(d\left(x_{i}, x_{j}\right) \leq \frac{1}{k+1}\right),
$$

where $[N, N+g(N)]$ denotes the set $\{N, N+1, \ldots, N+g(N)\}$. By examining the above form in conjunction with the logical metatheorems of proof mining, it can be seen that it fits into the required logical complexity and therefore one can extract from a classical proof of the statement a rate of metastability, i.e. a functional $\Phi: \mathbb{N} \times \mathbb{N}^{\mathbb{N}} \rightarrow \mathbb{N}$ satisfying

$$
\exists N \leq \Phi(k, g) \forall i, j \in[N, N+g(N)]\left(d\left(x_{i}, x_{j}\right) \leq \frac{1}{k+1}\right),
$$

for all $k \in \mathbb{N}$ and all $g: \mathbb{N} \rightarrow \mathbb{N}$. Such rates, even if they are not full rates of convergence, may still be deemed useful, for example in the further analysis of proofs of well-behaved statements which nevertheless use the convergence result as a premise.

Now, the proof of the second case of Theorem 1.1 consists essentially of an argument that crucially uses the property of the sequence generated by the algorithm being Fejér monotone with respect to the set of minimizers of the function. Such an argument has been analyzed before in a highly general form by Kohlenbach, the first author and Nicolae [12], an analysis that has been since applied to other similar instances $[13,16]$, therefore illustrating how modular the techniques of proof mining can become. An application of this idea to the proximal point algorithm in CAT( 0 ) spaces has been carried out in [17]. The goal of our analysis in Section 4 will be to show that one may use a way of approximating the minimizer set that captures more accurately the spirit of the problem in order to obtain, for complete totally bounded CAT(0) spaces, a rate of metastability for the sequence together with a precise finitization of the fact that the limit of the sequence is a minimizer of the function.

We finish the introduction by recalling some notions that are used for expressing our quantitative results. We denote $[m, n]=\{m, m+1, \ldots, n\}$ for all $m, n \in \mathbb{N}$ with $m \leq n$. For any $f: \mathbb{N} \rightarrow \mathbb{N}$, we define

$$
f^{M}: \mathbb{N} \rightarrow \mathbb{N}, \quad f^{M}(n)=\max _{i \leq n} f(i) .
$$

Then $f^{M}$ is nondecreasing and $f(n) \leq f^{M}(n)$ for all $n \in \mathbb{N}$.

Let $(X, d)$ be a metric space and $\left(a_{n}\right)$ be a convergent sequence in $X$ with $\lim _{n \rightarrow \infty} a_{n}=a$. A mapping $\varphi: \mathbb{N} \rightarrow \mathbb{N}$ is said to be:

(i) a rate of convergence of $\left(a_{n}\right)$ if for all $k \in \mathbb{N}$,

$$
d\left(a_{n}, a\right) \leq \frac{1}{k+1} \quad \text { for all } n \geq \varphi(k) .
$$

(ii) a Cauchy modulus of $\left(a_{n}\right)$ if for all $k \in \mathbb{N}$,

$$
d\left(a_{m}, a_{n}\right) \leq \frac{1}{k+1} \quad \text { for all } m, n \geq \varphi(k) .
$$

If $\left(b_{n}\right)$ is a sequence of nonnegative reals such that the series $\sum_{n=0}^{\infty} b_{n}$ diverges, then a rate of divergence of $\sum_{n=0}^{\infty} b_{n}$ is a mapping $\theta: \mathbb{N} \rightarrow \mathbb{N}$ satisfying

$$
\sum_{n=0}^{\theta(P)} b_{n} \geq P \quad \text { for all } P \in \mathbb{N} \text {. }
$$




\section{Preliminaries AND First Results}

We briefly recall some notions on geodesic spaces. Let $(X, d)$ be a metric space. A geodesic in $X$ is a map $\gamma:[a, b] \rightarrow X($ where $a, b \in \mathbb{R})$ satisfying

$$
d(\gamma(s), \gamma(t))=|s-t| \quad \text { for all } s, t \in[a, b] .
$$

A geodesic segment in $X$ is the image $\gamma([a, b])$ of a geodesic $\gamma:[a, b] \rightarrow X$. If we denote $x:=\gamma(a)$ and $y:=\gamma(b)$, then we say that the geodesic segment $\gamma([a, b])$ joins $x$ and $y$. The metric space $(X, d)$ is a (uniquely) geodesic space if every two distinct points are joined by a (unique) geodesic segment. One can easily see that $(X, d)$ is a uniquely geodesic space if and only if for any $x, y \in X$ there exists a unique geodesic $\gamma:[0, d(x, y)] \rightarrow X$ such that $\gamma(0)=x$ and $\gamma(d(x, y))=y$.

A CAT(0) space is a geodesic space $(X, d)$ satisfying the following: for all $z \in X$, all geodesics $\gamma$ : $[a, b] \rightarrow X$ and all $t \in[0,1]$

$$
d^{2}(z, \gamma((1-t) a+t b)) \leq(1-t) d^{2}(z, \gamma(a))+t d^{2}(z, \gamma(b))-t(1-t) d^{2}(\gamma(a), \gamma(b)) .
$$

It is a well-known fact that $\mathrm{CAT}(0)$ spaces are uniquely geodesic.

In the sequel, $(X, d)$ is a CAT(0) space. If $x, y \in X$ and $\gamma:[0, d(x, y)] \rightarrow X$ is the unique geodesic that joins them, we denote, for any $t \in[0,1]$, the point $\gamma(t d(x, y))$ by $(1-t) x+t y$. Thus,

$$
d(x,(1-t) x+t y)=t d(x, y) \quad \text { and } \quad d(y,(1-t) x+t y)=(1-t) d(x, y) .
$$

Using this notation, it is an immediate exercise to see that (2.1) can be written as follows: for all $x, y, z \in X$ and all $t \in[0,1]$,

$$
\left.d^{2}(z,(1-t) x+t y)\right) \leq(1-t) d^{2}(z, x)+t d^{2}(z, y)-t(1-t) d^{2}(x, y) .
$$

Let $f: X \rightarrow(-\infty,+\infty]$ be a convex, lower semicontinuous (lsc), proper function. We denote by $\operatorname{Argmin}(f)$ the set of minimizers of $f$ and we assume that $\operatorname{Argmin}(f)$ is nonempty. For any $\gamma>0$, the resolvent $J_{\gamma}$ of $f$ of order $\gamma>0$ [7] is defined as follows:

$$
J_{\gamma}: X \rightarrow X, \quad J_{\gamma}(x)=\operatorname{argmin}_{y \in X}\left[f(y)+\frac{1}{2 \gamma} d^{2}(x, y)\right] .
$$

We also say that $J_{\gamma}$ is the proximal mapping of order $\gamma$. As proved in [7], $J_{\gamma}$ is a nonexpansive mapping: for all $x, y \in X$,

$$
d\left(J_{\gamma} x, J_{\gamma} y\right) \leq d(x, y)
$$

If $T: X \rightarrow X$, we denote by $F i x(T)$ the set of fixed points of $T$. The definition of the proximal mapping is motivated by the following well-known result.

Proposition 2.1. For any $\gamma>0$, Fix $\left(J_{\gamma}\right)=\operatorname{Argmin}(f)$.

Let $\left(\gamma_{n}\right)$ be a sequence in $(0, \infty)$. The proximal point algorithm $\left(x_{n}\right)$ starting with $x \in X$ is defined as follows:

$$
x_{0}:=x, \quad x_{n+1}:=J_{\gamma_{n}} x_{n} \text { for all } n \in \mathbb{N} .
$$

It is easy to see that for all $n, l \in \mathbb{N}$ and all $q \in X$,

$$
d\left(x_{n+l}, q\right) \leq d\left(x_{n}, q\right)+\sum_{i=n}^{n+l-1} d\left(q, J_{\gamma_{i}} q\right) .
$$

As a consequence, we get that 
Lemma 2.1. $\left(x_{n}\right)$ is Fejér monotone with respect to Argmin $(f)$, that is

$$
d\left(x_{n+1}, p\right) \leq d\left(x_{n}, p\right)
$$

for all $p \in \operatorname{Argmin}(f)$ and all $n \in \mathbb{N}$.

We recall the following result that will be used in this paper.

Proposition 2.2. [17, Lemma 2.6.(ii)]

Let $b \in \mathbb{R}$ be such that $d(x, p) \leq b$ for some $p \in \operatorname{Argmin}(f)$. Assume that $\sum_{n=0}^{\infty} \gamma_{n}=\infty$ with rate of divergence $\theta$.

Then $\lim _{n \rightarrow \infty} f\left(x_{n}\right)=\min (f)$, with a (nondecreasing) rate of convergence

$$
\beta_{b, \theta}: \mathbb{N} \rightarrow \mathbb{N}, \quad \beta_{b, \theta}(k)=\theta^{M}\left(\left\lceil\frac{b^{2}(k+1)}{2}\right\rceil\right)+1 .
$$

We define, for any $k \in \mathbb{N}$ and any $\gamma>0$,

$$
\operatorname{Argmin}_{k}(\gamma f):=\left\{x \in X \mid \text { for all } y \in X, \gamma f(x) \leq \gamma f(y)+\frac{1}{k+1}\right\} .
$$

We call an element of $\operatorname{Argmin}_{k}(\gamma f)$ a $k$-approximate minimizer of $\gamma f$. In addition, we define a $k$ approximate fixed point of an operator $T: X \rightarrow X$ to be a point $x$ such that

$$
d(x, T x) \leq \frac{1}{k+1}
$$

The following result will be needed in Section 4.

\section{Proposition 2.3. Define}

$$
\psi: \mathbb{N} \rightarrow \mathbb{N}, \quad \psi(k)=2(k+1)^{2}-1 .
$$

Then, for all $\gamma>0$ and all $k \in \mathbb{N}$, any $\psi(k)$-approximate minimizer of $\gamma f$ is a $k$-approximate fixed point of $J_{\gamma}$.

Proof. Let $x$ be a $\psi(k)$-approximate minimizer of $\gamma f$. Then we have that

$$
\gamma f(x) \leq \gamma f\left(J_{\gamma} x\right)+\frac{1}{2(k+1)^{2}} .
$$

On the other hand, by the definition of $J_{\gamma}$,

$$
\gamma f\left(J_{\gamma} x\right)+\frac{1}{2} d^{2}\left(x, J_{\gamma} x\right) \leq \gamma f(x) .
$$

Putting these together, we immediately obtain that

$$
d\left(x, J_{\gamma} x\right) \leq \frac{1}{k+1} .
$$




\section{A RATE OF CONVERGENCE FOR UNIFORMLY CONVEX FUNCTIONS}

Let us recall that, if $(X, d)$ is a metric space, then a function $f: X \rightarrow(-\infty, \infty]$ is uniformly convex if there exists a nondecreasing function $\varphi:[0, \infty) \rightarrow[0, \infty]$ vanishing only at 0 such that for all $x, y \in X$ and all $t \in[0,1]$,

$$
f((1-t) x+t y) \leq(1-t) f(x)+t f(y)-t(1-t) \varphi(d(x, y)) .
$$

Such a function $\varphi$ is called a modulus of uniform convexity of $f$. We also say that $f$ is uniformly convex with modulus $\varphi$.

The main result of this section is the following effective version of Theorem 1.1 for uniformly convex functions.

Theorem 3.1. Let $X$ be a complete $C A T(0)$ space and $f: X \rightarrow(-\infty, \infty]$ be an lsc proper function with $\operatorname{Argmin}(f) \neq \emptyset$. Assume, furthermore, that $f$ is uniformly convex with modulus $\varphi$. Suppose that $x \in X$ and $b>0$ are such that $d(x, p) \leq b$ for some minimizer $p$ of $f$ and that $\left(\gamma_{n}\right)$ is a sequence in $(0, \infty)$ such that $\sum_{n=0}^{\infty} \gamma_{n}=\infty$ with rate of divergence $\theta$. Let $\left(x_{n}\right)$ be the proximal point algorithm starting with $x$. Define

$$
\Sigma_{b, \theta, \varphi}: \mathbb{N} \rightarrow \mathbb{N}, \quad \Sigma_{b, \theta, \varphi}(k)=\beta_{b, \theta}\left(\left\lceil\frac{8}{\varphi\left(\frac{1}{k+1}\right)}\right\rceil\right),
$$

with $\beta_{b, \theta}$ given by (2.5). Then $\Sigma_{b, \theta, \varphi}$ is a rate of convergence for $\left(x_{n}\right)$ and the limit of this sequence is a minimizer of $f$.

Proof. Let $k \in \mathbb{N}$ and $n, m \geq \Sigma_{b, \theta, \varphi}(k)$ be arbitrary. Applying (3.1) for $x_{n}, x_{m}$ and $\frac{1}{2}$, we get that

$$
f\left(\frac{x_{n}+x_{m}}{2}\right) \leq \frac{1}{2} f\left(x_{n}\right)+\frac{1}{2} f\left(x_{m}\right)-\frac{1}{4} \varphi\left(d\left(x_{n}, x_{m}\right)\right) .
$$

Hence

$$
\begin{aligned}
\frac{1}{4} \varphi\left(d\left(x_{n}, x_{m}\right)\right) & \leq \frac{1}{2} f\left(x_{n}\right)+\frac{1}{2} f\left(x_{m}\right)-f\left(\frac{x_{n}+x_{m}}{2}\right) \\
& \leq \frac{1}{2} f\left(x_{n}\right)+\frac{1}{2} f\left(x_{m}\right)-\min (f) .
\end{aligned}
$$

By Proposition 2.2, we have that $\lim _{n \rightarrow \infty} f\left(x_{n}\right)=\min (f)$, with rate of convergence $\beta_{b, \theta}$. Using the definition of $\Sigma_{b, \theta, \varphi}$, we get that

$$
f\left(x_{n}\right)-\min (f), f\left(x_{m}\right)-\min (f) \leq \frac{1}{\left\lceil\frac{8}{\varphi\left(\frac{1}{k+1}\right)}\right\rceil+1} .
$$

It follows that

$$
\frac{1}{4} \varphi\left(d\left(x_{n}, x_{m}\right)\right) \leq \frac{1}{\left\lceil\frac{8}{\varphi\left(\frac{1}{k+1}\right)}\right]+1} \leq \frac{1}{8} \varphi\left(\frac{1}{k+1}\right)<\frac{1}{4} \varphi\left(\frac{1}{k+1}\right) .
$$

Since $\varphi$ is nondecreasing, we must have

$$
d\left(x_{n}, x_{m}\right) \leq \frac{1}{k+1}
$$


Thus, we have proved that $\left(x_{n}\right)$ is Cauchy with $\Sigma_{b, \theta, \varphi}$ being a Cauchy modulus. Since $X$ is complete, $\left(x_{n}\right)$ is also convergent and let $z$ be its limit. Setting $m \rightarrow \infty$ in the above relation, we obtain that

$$
d\left(x_{n}, z\right) \leq \frac{1}{k+1}
$$

proving that $\Sigma_{b, \theta, \varphi}$ is indeed a rate of convergence for $\left(x_{n}\right)$.

Finally, by [2, Remark 1.7] (see Theorem 1.1), the limit $z$ of $\left(x_{n}\right)$ is a minimizer of $f$.

\section{A RATE OF METASTABility FOR TOTALLY BOUNDED SPACES}

Let $(X, d)$ be a metric space and $F \subseteq X$. The following notion, introduced in [12], is essential for this section: an approximation of $F$ is a family $\left(A F_{k}\right)_{k \in \mathbb{N}}$ of subsets of $X$ satisfying

$$
F=\bigcap_{k \in \mathbb{N}} A F_{k} \quad \text { and } \quad A F_{k+1} \subseteq A F_{k} \text { for all } k \in \mathbb{N} .
$$

Using such approximations, Kohlenbach, the first author and Nicolae developed in [12] very general methods that can be used to obtain quantitative forms, providing uniform rates of metastability, of strong convergence results in compact spaces for sequences $\left(x_{n}\right)$ satisfying a general form of Fejér monotonicity. These methods have been already applied to the proximal point algorithm: in [12] with $F$ being the set of zeros of a maximally monotone operator in a Hilbert space and in [17] for the setting from this paper. The approximation used in [17] for $F:=\operatorname{Argmin}(f)$ was defined as:

$$
A F_{k}:=\left\{x \in X \mid \text { for all } i \leq k, d\left(x, J_{\gamma_{i}} x\right) \leq \frac{1}{k+1}\right\} .
$$

In this paper, we use the following approximation of $F$, which most faithfully exhibits the meaning of the problem:

$$
A F_{k}:=\operatorname{Argmin}_{k}(f)=\left\{x \in X \mid \text { for all } y \in X, f(x) \leq f(y)+\frac{1}{k+1}\right\} .
$$

Obviously, $\operatorname{Argmin}_{k+1}(f) \subseteq \operatorname{Argmin}_{k}(f)$ for every $k \in \mathbb{N}$ and $\operatorname{Argmin}(f)=\bigcap_{k \in \mathbb{N}} \operatorname{Argmin}_{k}(f)$.

We recall $[5,12]$ that a modulus of total boundedness for a metric space $(X, d)$ is a function $\alpha: \mathbb{N} \rightarrow \mathbb{N}$ such that for any $k \in \mathbb{N}$ and any sequence $\left(x_{n}\right)$ in $X$,

$$
\text { there exist } i, j \in[0, \alpha(k)], i<j \text { such that } d\left(x_{i}, x_{j}\right) \leq \frac{1}{k+1} .
$$

It is an easy exercise to verify that $X$ is totally bounded if and only if it has a modulus of total boundedness. Furthermore, it is well-known that a metric space is compact if and only if it is complete and totally bounded.

The main result of this section is an effective version of Theorem 1.1 for totally bounded spaces.

Theorem 4.1. Let $X$ be a totally bounded CAT(0) space with modulus $\alpha$ and $f: X \rightarrow(-\infty, \infty]$ be a convex lsc proper function with $F:=\operatorname{Argmin}(f) \neq \emptyset$. Let $x \in X$ and $b>0$ be such that $d(x, p) \leq b$ for some minimizer $p$ of $f$.

Assume that $\left(\gamma_{n}\right)$ is a sequence in $(0, \infty)$ such that $\sum_{n=0}^{\infty} \gamma_{n}=\infty$ with rate of divergence $\theta$ and $L: \mathbb{N} \rightarrow \mathbb{N}$ is a nondecreasing mapping satisfying $L(k) \geq \max _{0 \leq i \leq k} \gamma_{i}$ for all $k \in \mathbb{N}$.

Let $\left(x_{n}\right)$ be the proximal point algorithm starting with $x$. Define $\Psi_{b, \theta, L, \alpha}$ and $\Omega_{b, \theta, L, \alpha}$ as in Table 1 . Then

(i) $\Psi_{b, \theta, L, \alpha}$ is a rate of metastability for $\left(x_{n}\right)$. 
(ii) for all $k \in \mathbb{N}$ and all $g: \mathbb{N} \rightarrow \mathbb{N}$ there exists $N \leq \Omega_{b, \theta, L, \alpha}(k, g)$ such that

$$
\forall i, j \in[N, N+g(N)]\left(d\left(x_{i}, x_{j}\right) \leq \frac{1}{k+1}\right) \text { and } \forall m \geq N\left(x_{m} \leq \min (f)+\frac{1}{k+1}\right) .
$$

Proof. In the following claims, we show that the necessary moduli can be computed in our setting. We refer to [12] for the terminology.

Claim 1: $\left(x_{n}\right)$ is uniformly Fejér monotone with respect to $F$ with modulus $\chi_{L}$ defined in Table 1, that is: for all $n, m, r \in \mathbb{N}$,

$$
q \in \operatorname{Argmin}_{\chi_{L}(n, m, r)}(f) \quad \text { implies } \quad d\left(x_{n+l}, q\right)<d\left(x_{n}, q\right)+\frac{1}{r+1} \text { for all } l \leq m .
$$

Proof of claim: Let $n, m, r \in \mathbb{N}, q$ as in the hypothesis and $l \leq m$. By (2.4), we have that

$$
d\left(x_{n+l}, q\right) \leq d\left(x_{n}, q\right)+\sum_{i=n}^{n+l-1} d\left(q, J_{\gamma_{i}} q\right) \leq d\left(x_{n}, q\right)+\sum_{i=n}^{n+m-1} d\left(q, J_{\gamma_{i}} q\right) .
$$

Let $i \in\{n, \ldots, n+m-1\}$. We get that

$$
\begin{aligned}
\gamma_{i} f(q) & \leq \gamma_{i} f(y)+\frac{\gamma_{i}}{\chi_{L}(n, m, r)+1} \quad \text { since } q \in \operatorname{Argmin}_{\chi_{L}(n, m, r)}(f) \\
& \leq \gamma_{i} f(y)+\frac{L(n+m-1)}{\chi_{L}(n, m, r)+1} \quad \text { by the definition of } L \\
& =\gamma_{i} f(y)+\frac{1}{2(m(r+1)+1)^{2}} \quad \text { by the definition of } \chi_{L} .
\end{aligned}
$$

Thus, $q$ is a $\psi(m(r+1))$-approximate fixed point of $\gamma_{i} f$, where $\psi$ is defined by (2.6). We can apply Proposition 2.3 for $\gamma_{i}$ to obtain that $q$ is an $m(r+1)$-approximate fixed point of $J_{\gamma_{i}}$, that is

$$
d\left(q, J_{\gamma_{i}} q\right) \leq \frac{1}{m(r+1)+1} .
$$

It follows that

$$
\sum_{i=n}^{n+m-1} d\left(q, J_{\gamma_{i}} q\right) \leq \frac{m}{m(r+1)+1}<\frac{1}{r+1}
$$

By (4.1), the claim follows.

Claim 2: $\left(x_{n}\right)$ is asymptotically regular with respect to $F$, with a rate of asymptotic regularity $\beta_{b, \theta}$ defined by (2.5), that is:

$$
\forall k \in \mathbb{N} \forall n \geq \beta_{b, \theta}(k)\left(x_{n} \in \operatorname{Argmin}_{k}(f)\right) .
$$

Proof of claim: Let $k \in \mathbb{N}$. Applying Proposition 2.2, we get that for all $n \geq \beta_{b, \theta}(k)$,

$$
f\left(x_{n}\right) \leq \min (f)+\frac{1}{k+1} \leq f(y)+\frac{1}{k+1} \quad \text { for all } y \in X .
$$

Claim 3: $\left(x_{n}\right)$ has $F$-approximate points, with $\beta_{b, \theta}$ being an approximate $F$-point bound, that is:

$$
\forall k \in \mathbb{N} \exists N \leq \beta_{b, \theta}(k)\left(x_{N} \in \operatorname{Argmin}_{k}(f)\right) .
$$

Proof of claim: It is an obvious consequence of the second claim.

Now we can finish the proof of the theorem. 
(i) We apply [12, Theorem 5.1], based on the first and the third claims. Using the notations from [12], $\alpha_{G}:=i d_{\mathbb{R}_{+}}, \beta_{H}:=i d_{\mathbb{R}_{+}}, \gamma:=\alpha$, hence $P=\alpha(4 k+3)$, and, furthermore,

$$
\begin{aligned}
& \chi_{g, L}(n, r)=\chi_{L}(n, g(n), r)=2 L(n+g(n)-1)(g(n)(r+1)+1)^{2}-1 \\
& \chi_{g, L}^{M}(n, r)=\max _{i \leq n} \chi_{g, L}(i, r)=\max _{i \leq n} 2 L(i+g(i)-1)(g(i)(r+1)+1)^{2}-1 .
\end{aligned}
$$

(ii) As pointed out in [12, p. 23] and detailed in [14] (the preprint version of [12]), we can get (ii) as an application of [14, Theorem 5.8.(ii) and Corollary 5.9], based on the second claim and the following facts:

(a) the rate of asymptotic regularity $\beta_{b, \theta}$ satisfies $\beta_{b, \theta}^{M}=\beta_{b, \theta}$.

(b) $L^{M}=L$ (as $L$ is nondecreasing), hence, using the notation from [14, Corollary 5.9], we have that $\chi_{L}^{M}=\chi_{L}$.

\begin{tabular}{|l|}
$\Psi_{b, \theta, L, \alpha}: \mathbb{N} \times \mathbb{N}^{\mathbb{N}} \rightarrow \mathbb{N}, \quad\left(\Psi_{0}\right)_{b, \theta, L}: \mathbb{N} \times \mathbb{N} \times \mathbb{N}^{\mathbb{N}} \rightarrow \mathbb{N}$ \\
$\Psi_{b, \theta, L, \alpha}(k, g)=\left(\Psi_{0}\right)_{b, \theta, L}(\alpha(4 k+3), k, g)$ \\
$\left(\Psi_{0}\right)_{b, \theta, L}(0, k, g)=0$ \\
$\left(\Psi_{0}\right)_{b, \theta, L}(n+1, k, g)=\beta_{b, \theta}\left(\chi_{g, L}^{M}\left(\left(\Psi_{0}\right)_{b, \theta, L}(n, k, g), 4 k+3\right)\right)$ \\
$\chi_{L}: \mathbb{N} \times \mathbb{N} \times \mathbb{N} \rightarrow \mathbb{N}, \quad \chi_{L}(n, m, r)=2 L(n+m-1)(m(r+1)+1)^{2}-1$ \\
$\chi_{g, L}^{M}: \mathbb{N} \times \mathbb{N} \rightarrow \mathbb{N}, \quad \chi_{g, L}^{M}(n, r)=\max _{i \leq n} 2 L(i+g(i)-1)(g(i)(r+1)+1)^{2}-1$ \\
$\Omega_{b, \theta, L, \alpha}: \mathbb{N} \times \mathbb{N}^{\mathbb{N}} \rightarrow \mathbb{N}, \quad \Omega_{b, \theta, L, \alpha}(k, g)=\Psi_{b, \theta, L, \alpha}^{*}\left(k, g_{\beta_{b, \theta}(k)}\right)+\beta_{b, \theta}(k)$ \\
$\Psi_{b, \theta, L, \alpha}^{*}: \mathbb{N} \times \mathbb{N}^{\mathbb{N}} \rightarrow \mathbb{N}, \quad \Psi_{b, \theta, L, \alpha}^{*}(k, g)=\Psi_{b, \theta, L, \alpha^{M}}(k, g)$ \\
$g_{l}: \mathbb{N} \rightarrow \mathbb{N}, \quad g_{l}(n)=g^{M}(n+l)+l$ \\
with $\beta_{b, \theta}$ given by $(2.5)$
\end{tabular}

TABLE 1. Definitions of $\Psi_{b, \theta, L, \alpha}$ and $\Omega_{b, \theta, L, \alpha}$

\section{REFERENCES}

[1] H. Bauschke, P. Combettes, Convex Analysis and Monotone Operator Theory in Hilbert Spaces, Second Edition, Springer, 2017.

[2] M. Bačák, The proximal point algorithm in metric spaces, Israel J. Math. 194 (2013), 689-701.

[3] H. Brézis, P. Lions, Produits infinis de résolvantes, Israel J. Math. 29 (1978), 329-345.

[4] E. M. Briseid, Logical aspects of rates of convergence in metric spaces, J. Symbolic Logic 74 (2009), 1401-1428.

[5] P. Gerhardy, Proof mining in topological dynamics, Notre Dame J. Form. Log. 49 (2008), 431-446.

[6] O. Güler, On the convergence of the proximal point algorithm for convex minimization, SIAM J. Control Optim. 29 (1991), 403-419.

[7] J. Jost, Convex functionals and generalized harmonic maps into spaces of non positive curvature, Comment. Math. Helv. 70 (1995), 659-673. 
[8] U. Kohlenbach, Applied proof theory: Proof interpretations and their use in mathematics, Springer Monographs in Mathematics, Springer, 2008.

[9] U. Kohlenbach, Recent progress in proof mining in nonlinear analysis, IFCoLog J. Logics Appl. 10 (2017), $3357-3406$.

[10] U. Kohlenbach, Proof-theoretic methods in nonlinear analysis, draft, 2017; to appear in Proceedings of the ICM2018.

[11] U. Kohlenbach, Theorie der majorisierbaren und stetigen Funktionale und ihre Anwendung bei der Extraktion von Schranken aus inkonstruktiven Beweisen: Effektive Eindeutigkeitsmodule bei besten Approximationen aus ineffektiven Beweisen, PhD Thesis, Frankfurt am Main, 1990.

[12] U. Kohlenbach, L. Leuştean, A. Nicolae, Quantitative results on Fejér monotone sequences, Commun. Contemp. Math. 20 (2018), 1750015.

[13] U. Kohlenbach, L. Leuştean, A. Nicolae, Quantitative results on Fejér monotone sequences, preprint version, arXiv:1412.5563 [math.LO], 2015.

[14] U. Kohlenbach, G. López-Acedo, A. Nicolae, Quantitative asymptotic regularity for the composition of two mappings, Optimization 66 (2017), 1291-1299.

[15] L. Leuştean, A. Nicolae, A. Sipoş, An abstract proximal point algorithm, J. Global Optim. 2018, https://doi.org/10.1007/s10898-018-0655-9.

[16] L. Leuştean, V. Radu, A. Sipoş, Quantitative results on the Ishikawa iteration of Lipschitz pseudo-contractions, J. Nonlinear Convex Anal. 17 (2016), 2277-2292.

[17] L. Leuştean, A. Sipoş, An application of proof mining to the proximal point algorithm in CAT(0) spaces, in: A. Bellow, C. Calude, T. Zamfirescu (Editors), Mathematics Almost Everywhere, In Memory of Solomon Marcus, World Scientific, 2018, 153-168.

[18] B. Martinet, Régularisation d'inéquations variationnelles par approximations successives, Rev. Française Informat. Recherche Opérationnelle 4 (1970), 154-158.

[19] R. T. Rockafellar, Monotone operators and the proximal point algorithm, SIAM J. Control Optim. 14 (1976), 877-898.

[20] T. Tao, Soft analysis, hard analysis, and the finite convergence principle, Essay posted May 23, 2007, appeared in: T. Tao, Structure and Randomness: Pages from Year One of a Mathematical Blog, Amer. Math. Soc. 2008.

[21] T. Tao, Norm convergence of multiple ergodic averages for commuting transformations, Ergodic Theory Dynam. Systems 28 (2008), 657-688. 